Arq. Bras. Med. Vet. Zootec., v.63, n.2, p.317-321, 2011

\title{
Eletrocardiografia computadorizada em cães da raça Beagle
}

\author{
[Computerised electrocardiograph in Beagle dogs] \\ F.N. Gava ${ }^{1}$, D. Paulino-Junior ${ }^{1}$, G.B. Pereira-Neto ${ }^{1}$, J.P.E. Pascon ${ }^{1}$, M.G. Sousa ${ }^{1}$, \\ T. Chanpion ${ }^{1}$, A.A. Camacho ${ }^{2 *}$. \\ ${ }^{1}$ Aluno de Pós-graduação - Faculdade de Ciências Agrárias e Veterinárias - Unesp - Jaboticabal, SP \\ ${ }^{2}$ Faculdade de Ciências Agrárias e Veterinárias (FCAV) - Unesp - Campus de Jaboticabal \\ Via de Acesso Prof. Paulo Donato Castellane, s/n \\ 32092-600 - Jaboticabal, SP
}

\begin{abstract}
RESUMO
Estudaram-se os parâmetros eletrocardiográficos utilizando-se os métodos convencional, computadorizado e computadorizado impresso de 30 cães da raça Beagle clinicamente normais. As medidas eletrocardiográficas dos três diferentes traçados foram analisadas na derivação DII. A comparação entre os métodos revelou diferença nos valores da onda $\mathrm{P}$, onda R, complexo QRS, intervalo QT e intervalo PR e na polaridade da onda T. Este fato deve ser levado em consideração, na dependência do método a ser utilizado.
\end{abstract}

Palavras-chave: cão, eletrocardiograma

\begin{abstract}
Electrocardiographic parameters were studied in thirty healthy adult Beagles, using conventional and computerized methods and reading the computerized registration printed on paper. The electrocardiographic measurements of the three different tracings were analyzed in lead II. The results obtained showed that there are differences among the three methods for $P$ wave, $R$ wave, QRS complex, $Q T$ interval, PR interval and polarity of the T wave. Special attention should be given to these differences, depending on the chosen method.
\end{abstract}

Keywords: dog, electrocardiogram

\section{INTRODUÇÃO}

A eletrocardiografia (ECG) é um exame eficaz em medicina veterinária e de obtenção relativamente fácil. Esse recurso tem sido utilizado para diagnóstico da maioria das arritmias, pois determina o ritmo e a frequência cardíaca, fornecendo informações a respeito do miocárdio, por meio das deflexões P-QRS-T no traçado eletrocardiográfico. Alterações nessas deflexões são consequências de estados patológicos ou fisiológicos do coração e outros órgãos, e as informações obtidas por meio da ECG são essenciais para a determinação do tipo, origem e severidade das arritmias, bem como do

Recebido em 9 de novembro de 2009

Aceito em 17 de março de 2011

*Autor para correspondência (corresponding author)

E-mail: camacho@fcav.unesp.br direcionamento terapêutico (Edwards, 1987; Tilley, 1992; Fulop et al., 2006; Ferreira et al., 2008).

Uma variação do método de representação da atividade elétrica do coração que surgiu com o avanço da informática é a eletrocardiografia computadorizada. Essa vem sendo cada vez mais empregada na medicina veterinária, devido ao fato de apresentar maior exatidão, possibilidade de utilização em serviços eletrocardiográficos de emergência, menor tempo requerido para o exame e capacidade de realizar grandes quantidades de eletrocardiogramas em menor tempo (Tilley, 1992; Shiwen et al., 1996; Nunes 2002; Souza et al., 2002). Assim, dados sobre esse exame em cães necessitam de estudos complementares (Skoula e Gordon, 1996; Wolf et al., 2000). 
Já foram demonstradas diferenças entre valores obtidos por meio da ECG convencional com a computadorizada em cães e gatos (Wolf et al., 2000; Camacho et al., 2010), em que os autores ressaltaram a importância desse fato no momento da interpretação dos exames eletrocardiográficos para animais clinicamente normais e cardiopatas.

Sabe-se que cães da raça Beagle são muito utilizados em várias investigações científicas, desse modo, pesquisas com eletrocardiografia computadorizada e padronização para essa raça são de extrema valia (Vandenhende, 2001; Heerdt et al., 2004). Com isso, o objetivo deste trabalho foi analisar e comparar os registros eletrocardiográficos em cães da raça Beagle, não anestesiados, obtidos pelos métodos convencional, computadorizado e pela leitura direta do registro computadorizado impresso, padronizando os valores obtidos informaticamente.

\section{MATERIAL E MÉTODOS}

Foram utilizados 30 cães da raça Beagle adultos, entre machos e fêmeas, clinicamente normais, com pesos corporais entre 9 a $17 \mathrm{~kg}$, mantidos em canis apropriados, com ração e água ad libitum. A triagem foi realizada na seleção dos animais, por meio de exames físico, radiográfico, eletrocardiográfico, ecodopplercardiográfico e hematológicos (hemograma e bioquímica sérica).

Para a eletrocardiografia convencional, foi utilizado um aparelho monocanal (ECG 6 ECAFIX) munido de papel termossensível milimetrado próprio para traçados eletrocardiográficos. Para os registros eletrocardiográficos pelo método informatizado, foi utilizado um aparelho eletrocardiográfico computadorizado (Módulo de Aquisição de ECG para Computador (ECG-PC versão 2.07) ${ }^{\circledR}$ Tecnologia Eletrônica Brasileira (TEB)).

No momento do exame eletrocardiográfico, os animais foram mantidos em decúbito lateral direito, fixando-se os eletrodos (Becton Dickinson B-D). Ambos os registros, ECG convencional e ECG computadorizada, foram realizados sequencialmente em cada cão, a partir das derivações bipolares DI, DII e DIII; unipolares aVR, aVL e aVF. Para as derivações de membros bipolares e unipolares aumentadas, os eletrodos torácicos direito e esquerdo foram posicionados acima do olécrano, e os eletrodos pélvicos direito e esquerdo acima dos ligamentos patelares no aspecto anterior de cada membro. A velocidade utilizada nos registros foi de $50 \mathrm{~mm}$ por segundo, com calibração da voltagem de 1 milivolte para cada centímetro $(1 \mathrm{mV}=1 \mathrm{~cm})$.

Após a realização do exame eletrocardiográfico pelo método computadorizado, foram obtidos traçados impressos dos trechos registrados em cada animal, para leitura dos parâmetros eletrocardiográficos em papel. Esse papel impresso possui milimetragem para leitura das ondas, intervalos e segmentos definidos pelo programa eletrônico.

As medidas eletrocardiográficas foram analisadas segundo a descrição feita por Tilley (1992), apenas na derivação bipolar II (DII), na qual se observaram as características do ritmo cardíaco e os valores referentes à frequência cardíaca, duração em milissegundos (ms) e amplitude em milivolts ( $\mathrm{mV}$ ) da onda P, duração do intervalo PR e do complexo QRS, amplitude da onda $\mathrm{R}$, duração do intervalo $\mathrm{QT}$, características da polaridade da onda $\mathrm{T}$ e presença ou não de desnivelamento do segmento ST. A determinação do eixo elétrico cardíaco médio foi expressa em graus, obtido a partir das derivações DI e DIII.

Os valores obtidos foram submetidos à análise de variância e, posteriormente, comparados pelo teste Dunnet. Para as características da onda T, segmento ST e ritmo cardíaco, foi aplicado o teste do qui-quadrado considerando-se $\mathrm{P}<0,05$ como significativo.

\section{RESULTADOS E DISCUSSÃO}

Os traçados obtidos a partir dos três diferentes métodos podem ser observados na Fig. 1. Não houve diferença significativa entre os métodos para os parâmetros frequência cardíaca (FC) e eixo elétrico cardíaco. A duração da onda $\mathrm{P}$, do complexo QRS e do intervalo QT diferiu entre os três métodos. Os valores do intervalo PR foram significativamente mais altos somente no método computadorizado em relação ao método computadorizado impresso. Diferenças entre os métodos também foram observadas nas comparações das médias de milivoltagem da onda P e da onda R, em que estas apresentaramse significativamente mais elevadas nos métodos computadorizado e computadorizado impresso em relação ao método convencional (Tab. 1). 

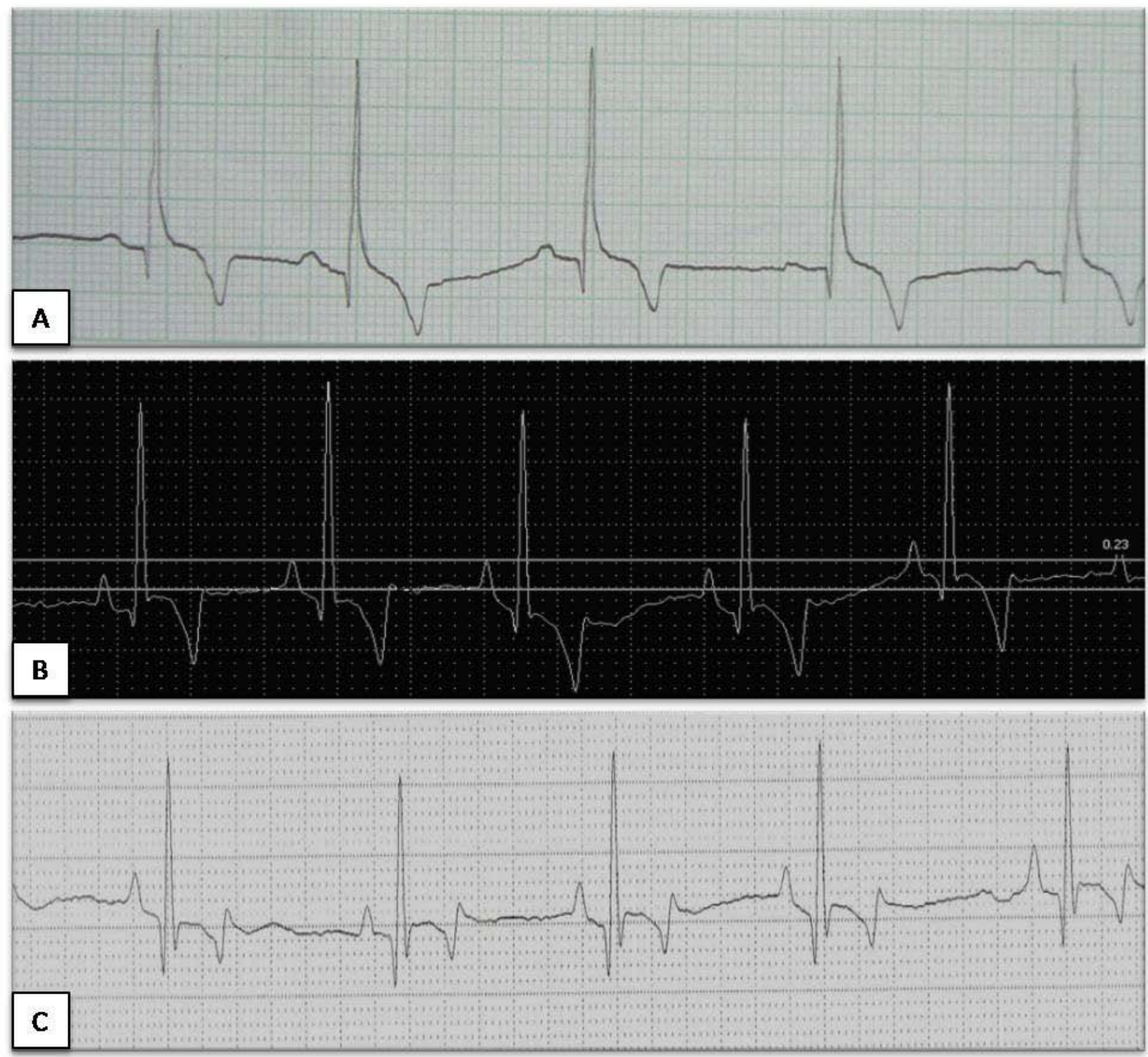

Figura 1. Cão. Imagem de trecho eletrocardiográfico convencional (A), computadorizado (B) e computadorizado impresso (C). Derivação DII, velocidade $50 \mathrm{~mm} /$ segundo, sensibilidade $\mathrm{N}$.

Tabela 1. Valores médios e desvios-padrão dos parâmetros eletrocardiográficos analisados por meio dos métodos eletrocardiográficos convencional, computadorizado e computadorizado impresso em cães da raça Beagle

\begin{tabular}{|c|c|c|c|}
\hline $\begin{array}{c}\text { Parâmetro } \\
\text { eletrocardiográfico }\end{array}$ & $\begin{array}{c}\text { Método } \\
\text { convencional }\end{array}$ & $\begin{array}{c}\text { Método } \\
\text { computadorizado }\end{array}$ & $\begin{array}{c}\text { Método } \\
\text { computadorizado } \\
\text { impresso } \\
\end{array}$ \\
\hline FC(bpm) & $126,66 \pm 21,22 a$ & $124,16 \pm 20,02 a$ & $124 \pm 19,93 a$ \\
\hline $\mathrm{P}(\mathrm{ms})$ & $29,66 \pm 8,08 \mathrm{c}$ & $46,83 \pm 4,19 a$ & $37,26 \pm 4,94 b$ \\
\hline $\mathrm{P}(\mathrm{mV})$ & $0,17 \pm 0,04 b$ & $0,25 \pm 0,06 a$ & $0,23 \pm 0,05 a$ \\
\hline $\mathrm{PR}(\mathrm{ms})$ & $91,66 \pm 11,47 a b$ & $94,03 \pm 12,07$ a & $88,83 \pm 11,27 b$ \\
\hline QRS(ms) & $44,00 \pm 11,62 c$ & $64,80 \pm 9,91 \mathrm{a}$ & $56,93 \pm 9,1 b$ \\
\hline $\mathrm{R}(\mathrm{mV})$ & $1,71 \pm 0,49 a$ & $1,49 \pm 0,60 b$ & $1,57 \pm 0,53 b$ \\
\hline QT(ms) & $185,33 \pm 13,57 \mathrm{c}$ & $199,56 \pm 15,97 a$ & $191,33 \pm 15,25 b$ \\
\hline Eixo(graus) & $73,26 \pm 15,24 a$ & $70,36 \pm 18,37 a$ & $70,56 \pm 16,15 \mathrm{a}$ \\
\hline
\end{tabular}

FC: frequência cardíaca; bpm: batimentos por minuto; ms: milissegundos; mV: milivolts. Letras distintas na mesma linha indicam diferenças significativas entre os métodos. 
Em relação à polaridade da onda $\mathrm{T}$, também houve diferença entre os métodos estudados (Tab. 2). Foi encontrada menor porcentagem de ondas $\mathrm{T}$ bifásicas no método convencional. Em ambos os métodos, houve predominância de segmento ST isoelétrico, porém foi encontrada porcentagem maior de infradesnivelamento na eletrocardiografia computadorizada em relação aos outros métodos (Tab. 3). Não houve diferença quanto ao ritmo cardíaco, e houve prevalência de arritmia sinusal respiratória (80\%) nos três métodos utilizados (Tab. 4).

Tabela 2. Valores percentuais de ocorrência de ondas T, segundo sua polaridade, registrados por meio dos métodos eletrocardiográficos convencional, computadorizado e computadorizado impresso em cães da raça Beagle

\begin{tabular}{lccc}
\hline \multicolumn{1}{c}{ Método } & $\begin{array}{c}\text { Positiva } \\
(\%)\end{array}$ & $\begin{array}{c}\text { Negativa } \\
(\%)\end{array}$ & $\begin{array}{c}\text { Bifásica } \\
(\%)\end{array}$ \\
\hline ECG convencional & $16,7 \mathrm{a}$ & $63,3 \mathrm{a}$ & $20 \mathrm{a}$ \\
ECG computadorizada & $10 \mathrm{a}$ & $36,6 \mathrm{~b}$ & $53,4 \mathrm{~b}$ \\
ECG computadorizada & $13,3 \mathrm{a}$ & $33,3 \mathrm{~b}$ & $53,4 \mathrm{~b}$ \\
impressa & & & \\
\hline
\end{tabular}

ECG= eletrocardiografia. Letras diferentes na coluna indicam diferença significativa entre as porcentagens.

Tabela 3. Segmento ST: valores percentuais de ocorrência de supradesnivelamento (ST>0,15mV), ST isoelétrico e infradesnivelamento $(\mathrm{ST}>0,2 \mathrm{mV}$ ), registrados por meio dos métodos eletrocardiográficos convencional, computadorizado e computadorizado impresso em cães da raça Beagle

\begin{tabular}{lccc}
\multicolumn{1}{c}{ Método } & $\begin{array}{c}\text { Infradesnivelamento } \\
(\%)\end{array}$ & $\begin{array}{c}\text { Isoelétrico } \\
(\%)\end{array}$ & $\begin{array}{c}\text { Supradesnivelamento } \\
(\%)\end{array}$ \\
\hline ECG convencional & $6,6 \mathrm{a}$ & $76,7 \mathrm{a}$ & $16,7 \mathrm{a}$ \\
ECG computadorizada & $16,7 \mathrm{a}$ & $73,3 \mathrm{a}$ & $10 \mathrm{a}$ \\
ECG computadorizada impressa & $13,3 \mathrm{a}$ & $70 \mathrm{a}$ & $16,7 \mathrm{a}$ \\
\hline
\end{tabular}

ECG= eletrocardiografia. Letras diferentes na coluna indicam diferença significativa entre as porcentagens.

Tabela 4. Valores percentuais de ocorrência de ritmo sinusal e arritmia sinusal respiratória registrados por meio dos métodos eletrocardiográficos convencional, computadorizado e computadorizado impresso em cães da raça Beagle

\begin{tabular}{lcc}
\multicolumn{1}{c}{ Método } & $\begin{array}{c}\text { Ritmo sinusal } \\
(\%)\end{array}$ & $\begin{array}{c}\text { Arritmia sinusal } \\
\text { (\%) respiratória }\end{array}$ \\
\hline ECG convencional & $20 \mathrm{a}$ & $80 \mathrm{a}$ \\
ECG computadorizada & $20 \mathrm{a}$ & $80 \mathrm{a}$ \\
ECG computadorizada impressa & $20 \mathrm{a}$ & $80 \mathrm{a}$ \\
\hline
\end{tabular}

ECG= eletrocardiografia. Letras diferentes na coluna indicam diferença significativa entre as porcentagens.

As médias de todas as variáveis obtidas pelo método convencional confirmam outros resultados já descritos para eletrocardiografia em cães da raça Beagle (Noel, 1969; Osborne e Leach, 1971; Vandenhende, 2001; Heerdt et al., 2004; Hanton e Rabemampianina, 2006). Porém, isso não ocorreu ao se comparar os valores obtidos pelo método computadorizado. Essas diferenças encontradas entre as leituras corroboram os achados por Wolf et al. (2000), em cães, e também por Camacho et al. (2010), em felinos. Possivelmente, devem-se ao fato de o ser humano possuir menor capacidade de percepção para a leitura do que o computador.
Pelter et al. (1997) descreveram que o homem só consegue mensurar desníveis a partir de 0,05 milivolts, enquanto o computador detecta a partir de 0,01 milivolts.

Segundo Ware (2006), alterações das ondas do ECG (em amplitude ou duração) e dos segmentos observados podem sugerir aumento ou distúrbio de condução de uma câmara cardíaca em particular. Assim, no momento da interpretação do exame eletrocardiográfico, os valores basais para tais parâmetros devem estar corretamente estabelecidos dependendo do método a ser utilizado. 


\section{CONCLUSÕES}

Considerando-se que os três métodos eletrocardiográficos estudados são atualmente utilizados na rotina de cardiologia veterinária, deve-se atentar para as diferenças entre eles, principalmente para o método computadorizado em relação aos demais, em que os valores dos parâmetros analisados apresentaram-se acima dos valores de normalidade descritos para o método convencional. Assim, o profissional deve consultar valores preestabelecidos de acordo com o método a ser utilizado.

\section{AGRADECIMENTOS}

Os autores agradecem à Fundação de Amparo à Pesquisa do Estado de São Paulo (FAPESP), pelo apoio financeiro.

\section{REFERÊNCIAS BIBLIOGRÁFICAS}

CAMACHO, A.A.; PAULINO JR, D.; PASCON, J.P.E. et al. Comparison between conventional and computerized electrocardiography in cats. Arq. Bras. Med. Vet. Zootec., v.62, p.765-769, 2010.

EDWARDS, N.J. Bolton's handbook of canine and feline electrocardiography. 2..ed. Philadelphia: W.B. Saunders, 1987. 381p.

FERREIRA, F.S.; VALE, D.F.; RAMOS, R.M. et al. Eletrocardiograma na monitoração anestésico-cirúrgica de cães. Rev. Bras. Cienc. Anim., v.1, p.121-134, 2008

FULOP, L.; BANYASZ, T.; SZABO, G. et al. Effects of sex hormones on ECG parameters and expression of cardiac ion channels in dogs. Acta Physiol., v.188, p.163-171, 2006.

HANTON, G.; RABEMAMPIANINA, Y. The electrocardiogram of the Beagle dog: reference values and effect of sex, genetic strain, body position and heart rate. Lab. Anim., v.40, p. 123136, 2006

HEERDT, P.M.; KANG, R.B.S; ANDREW, B.S. et al. Cardiopulmonary effects of the novel neuromuscular blocking drug GW280430A (AV430A) in Dogs. Anestesiology, v. 100, p.846-851, 2004.
NOEL, P.R.B. The challenge of selecting the suitable animal species in toxicology. Nonrodents (particularly the dog). In: BARKER, C.; TRIPOD, J. The problems of species difference and statistics in toxicology. Amsterdam: Excerpta Medica Foundation, 1969. 157p.

NUNES, N. Monitoração da anestesia. In: FANTONI, D.T.; CORTOPASI, S.R.G. Anestesia em cães e gatos. São Paulo: Roca, 2002. p.15-22.

OSBORNE, B.E.; LEACH, G.D.H. The Beagle electrocardiogram. Food Cosmet. Toxicol., v.9, p.857- 864, 1971.

PELTER, M.M.; ADAMS, M.G.; DREW, B.J. Computer versus manual measurement of STsegment deviation. J. Electrocardiol., v.30, p.151-156, 1997.

SCHIWEN, Y.; WOHLFART, B.; OLSSON, S.B. et al. Clinical application of a microcomputer system for analysis of monophasic action potentials. PACE, v.19, p.297-308, 1996.

SKOULA, C.M.; GORDON, B.E. Retrospective clinical study of computer detected ECG abnormalities in a random source hound population. Lab. Anim., v.46, p.462-463, 1996.

SOUZA, A.P.; CARARETO, R.; NUNES, N. et al. Eletrocardiografia em cães anestesiados com cetamina-s ou cetamina. Cienc. Rural, v.32, p.787-791, 2002.

TILLEY, L.P. Essentials of canine and feline electrocardiography. 3.ed. Philadelphia: Lea \& Febiger, 1992. 470p.

VANDENHENDE, F. Heart rate-specific reference ranges for QT-Interval in beagle dogs. Drug Inf. J., v.35, p.1179-1188, 2001.

WARE, W.A., Exames diagnósticos do sistema cardiovascular. In: NELSON, R.W.; COUTO, C.G. Medicina interna de pequenos animais. 3.ed. Rio de Janeiro: Elsevier, 2006. cap.2, p.1-16.

WOLF, R.; CAMACHO, A.A.; SOUZA, R.C.A. Eletrocardiografia computadorizada em cães. Arq. Bras. Med. Vet. Zootec., v.52, p.610-615, 2000. 